\title{
La Calidad del Servicio Personalizado en la Gestión Hotelera
}

Quality in Customized Service in Hotel Manaǵement

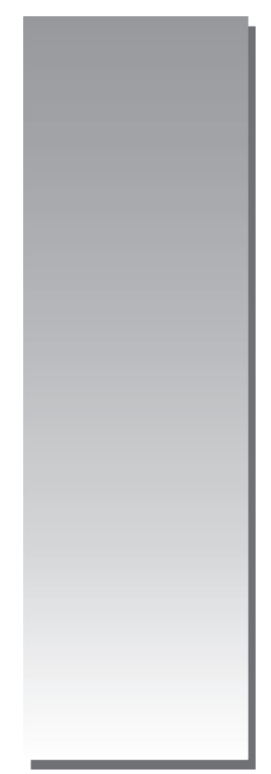

Dr. Ricardo Rolando Fonseca Saldaña" rfonseca20@yahoo.es

Dr. Adm. Adán Humberto Estela Estela" adanestela@yahoo.com

[RECEPCIÓN: AGOSTO 2016 / CONFORMIDAD: OCTUBRE 2016]

\begin{abstract}
RESUMEN
El estudio comprende: personalización de servicios, gestión hotelera que influye en rentabilidad. Investigación exploratoria, descriptiva; diseño transversal descriptivo; muestra de 24 empresas hoteleras de la ciudad de Lima, 384 clientes entrevistados al momento de cancelar. Instrumento utilizado: cuestionario de 21 ítems, comprobándose que la personalización de los servicios está relacionada con la gestión hotelera basada en tres dimensiones: cultura de servicio personalizado, rentabilidad y competitividad. Para el contraste, se usó la prueba de hipótesis de chi cuadrado. Concluye que la personalización de los servicios asegura mejor satisfacción del cliente y alta rentabilidad a la empresa hotelera. El factor humano es determinante en la aplicación de servicio personalizado. Los mecanismos que contribuyen a personalizar los servicios hoteleros son: búsqueda de aumento de ventas y el pedido del cliente es la razón básica de la personalización de servicios.
\end{abstract}

Palabras clave: personalización de servicios, cultura de servicio personalizado, rentabilidad, y competitividad

\section{ABSTRACT}

The research consists of: the customization of services, the influence of hotel management in profitability. Exploratory and descriptive research; sample of 24 hotel companies in the city of Lima, 384 customers were interviewed when paying. Instrument used: 21-item questionnaire. It was confirmed that the customization of services is related to hotel management based on three dimensions: Culture of Customized Service, Profitability and Competitiveness. For this contrast, the Chi-square hypothesis test was used. The research concludes that the customization of services ensures a better customer satisfaction and a high return to the hotel company. The human factor is crucial when implementing a customized service. The mechanisms contributing to customize hotel services are: searching of increasing sales and that customers' order which is the main reason for customizing the services.

Key words: Customization of service; Culture of Customized Service, Profitability and Competitiveness.

\footnotetext{
* Profesor Asociado de la Facultad de Ciencias Administrativas Universidad Nacional Mayor de San Marcos

** Profesor Asociado Contratado

Universidad Ricardo Palma
} 


\section{INTRODUCCIÓN}

La gestión turística percibe cambios en estructuras y procesos empresariales, impulsados por consumidores exigentes. Nuevos competidores con recursos tecnológicos y habilidades profesionales que definen nuevas reglas de juego para los agentes económicos internos y externos, que facilitan respuestas competitivas a demandas minuciosas. El propósito de la investigación pretende validar las experiencias exitosas presentadas en términos de mejores prácticas, propiciando que el impacto económico y sociocultural en el sector hotelero sea trascendente para el país; determinando la influencia de la personalización de los servicios en las preferencias de los clientes, el incremento de las ventas y la rentabilidad; el entorno social en el cual desarrolla su actividad económica; además de la relación de la cultura del buen servicio con la competitividad de la industria hotelera en el Perú.

El crecimiento observado en estadísticas relacionadas con el turismo motiva alta competencia a quienes han implementado una estrategia competitiva basada en la personalización del servicio que asegura la satisfacción del cliente; por ende, rentabilidad para la empresa hotelera. Los autores Czinkota \& Ronkainen (2007) afirman que el crecimiento del sector servicio es un fenómeno global y los servicios contribuyen en promedio con más del 60\% del PBI de todos los países.

El sector turístico, conocido como "industria global sin chimenea", presenta la necesidad de elaborar el "paquete turístico perfecto", que debe constar de paisajes hermosos, infraestructura hotelera, misterios arqueológicos, transporte cómodo, instalaciones de comida, comunicaciones y el factor humano, que es el eje que articula y activa la acción de este circuito. A nivel mundial el turismo contribuye al desarrollo de los países, otorgando divisas al Estado y generando un efecto multiplicador que dinamiza toda la economía; sin embargo, no todos los países tienen la opción de contar con atractivos turísticos naturales y arqueológicos como el Perú, con una geografía marcada por tres regiones naturales y cuyos atractivos turísticos y arqueológicos son reconocidos con la condición de patrimonio cultural de la humanidad.

El estudio contribuye a generar una tendencia de cambio que atraiga a más personas a trabajar en este sector. El fortalecimiento de la imagen internacional del país ha generado una corriente de expectativas de negocios en el Perú de grandes inversionistas, además de turistas ávidos de conocer las riquezas culturales, naturales y espirituales pasadas y presentes, legado de nuestra cultura incaica.

En estos últimos años hemos observado un crecimiento en las estadísticas de afluencia de turistas, construcción de hoteles, restaurantes para turistas, nuevos centros educativos de turismo, agencias de viaje, líneas aéreas globalizadas; generando mayores inversiones en este sector, reflejado en la economía de nuestro país. La formación y la capacitación del personal en la aplicación de una estrategia de personalización de servicios es un factor definitivo. Es la persona quien debe aprender y aplicar todas las nuevas prácticas y conocimientos frescos que necesita, el mercado; ya que un personal entrenado adecuadamente será fuente de ventaja competitiva.

Porter (2010) sostiene que la creciente competencia internacional en el sector de servicios no es nueva. Multitud de compañías compiten internacionalmente en: transporte, seguros, turismo y otras actividades de servicios desde inicios del siglo XX. Este mismo autor señala que los EE.UU. fue el primer país en desarrollar el negocio de los servicios de alojamiento, país que mantiene clara ventaja competitiva.

Horovitz (1990) asevera que el servicio es el conjunto de tareas que el cliente espera, además del producto o servicio básico, como consecuencia del precio, la imagen y la reputación del mismo. El término servicio no debe confundirse con amabilidad y gentileza, siendo un complemento del valor añadido, pero no significa todo. Un servicio de calidad no se puede producir en una fábrica, ni entregar completo al cliente. Para ello, son los clientes/usuarios quienes acceden a la "fábrica". Por ejemplo, un restaurante, un hotel, etc.; para consumir el servicio a medida que se produce.

Según Kotler (2005), un servicio es cualquier actividad o beneficio que una parte puede ofrecer a otra, es esencialmente intangible y no resulta en la propiedad de nada. Su producción podría o no estar vinculada a un producto físico. Por ejemplo, rentar o alquilar una habitación en un hotel, viajar en avión, alquilar un auto, etc. Todas estas actividades implican la adquisición de un servicio.

La importancia de esta investigación permite determinar las cualidades del servicio, así como la innovación de los servicios; conceptualización, 
características y clasificaciones; el conocimiento del cliente, sus expectativas, la importancia del personal en la actividad hotelera, las perspectivas, gestión de personal, elemento importante en una estrategia de personalización de los servicios; la calidad y la personalización de los servicios y gestión de la calidad en la actividad hotelera y los elementos básicos para aplicar una buena personalización de servicios como estrategia competitiva de este sector importante en la economía peruana.

\section{CALIDAD Y PERSONALIZACIÓN DE SERVICIOS EN LA GESTIÓN HOTELERA}

En la actualidad para lograr el éxito, o para sobrevivir, las empresas necesitan practicar una nueva filosofía, basada en el conocimiento y la satisfacción de las necesidades de los clientes. Para lograrlo, "es necesario que toda la organización desarrolle y practique un sistema de entrega de valor al cliente que sea superior al de la competencia" (Kotler, 2005). Para la buena marcha de la empresa son tres: plazo, costo y calidad. Tradicionalmente, solo las dos primeras han sido objeto de atención aplicando una serie de técnicas. El nuevo enfoque afianza la calidad.

La calidad actúa como elemento de motivación, integración y satisfacción para los trabajadores, haciéndolos partícipes de los objetivos de la empresa. Es otra cultura, otra forma de pensar y actuar. Para Gallego (1976), la calidad es una forma de vida, una filosofía, una manera de entender este nuevo mundo. Hablar de calidad supone hablar de personas con actitudes y aptitudes que responden a las necesidades de esta sociedad global que busca respuestas que cubran expectativas de satisfacción individual y colectiva. El primer beneficiado de la calidad no es el producto o servicio, sino el ser humano. La práctica de esta filosofía supone, primero, sentir una satisfacción al informar, alojar y alimentar al consumidor, consiguiendo la satisfacción del cliente. De esta manera, se contribuye al bienestar de la humanidad.

Actualmente, la nueva política de calidad que impera en el mundo implica: Mejora del diseño, que se traduce en un gran esfuerzo hacia los métodos de calidad preventivos. Implantación del concepto cero defectos (Crosby), que consiste en hacer las cosas bien a la primera. Espíritu de mejora permanente y búsqueda de la optimización en productividad, eficacia y ahorro. A estos elementos que se refieren a la estrategia empresarial, hay que sumar otros, innatos a la cultura japonesa. Para Udaondo (1992), son: amor a su empresa, integración en su trabajo, Disciplina, espíritu de mejora, que se traduce en una elevada motivación. La práctica de estos elementos sumados a los anteriormente mencionados hace posible no solo la implantación de la calidad en una empresa, sino una gestión exitosa de la misma.

\section{FACTORES CLAVE Y DONES DE LA GESTIÓN DE LA CALIDAD DEL SERVICIO}

Un estudio del sector de hoteles de Andersen (1995), para identificar los factores clave de la calidad del sector, dio como resultado los siguientes factores: el concepto de "vacaciones totales", creando la infraestructura necesaria en el hotel para poder atender cualquier necesidad que pueda plantearse al cliente sin tener que salir del hotel: servicio médico, todo tipo de deportes en interior o al aire libre, distintos tipos de restaurantes, bares con diferentes ambientes, discotecas, etc. El concepto de "todo incluido" (all inclusive), es decir, cualquier servicio que pueda requerir el cliente está a su disposición sin cargo complementario. El objetivo es poder incrementar el precio de venta final del paquete y asegurarse ingresos que, de otro modo, podrían perderse para el hotel debido a la oferta complementaria que puede existir en la zona.

Algunos beneficios de un servicio de calidad. Conservación de los clientes: Estudios han demostrado que cuesta de cuatro a seis veces más crear a un cliente que conservar a uno ya existente. Un cliente satisfecho también recomendará el producto servicio a otras personas. Se sabe, en promedio, que un cliente satisfecho comunica sus experiencias a otras cinco personas. Por otro lado, un cliente insatisfecho transmite su falta de conformidad a diez o más personas. El hotel que logre satisfacer con calidad la necesidad de servicio de su cliente obtendrá una buena reputación. Conservar a los mejores empleados: La tendencia actual de los empleados es trabajar en organizaciones bien dirigidas y que oferten productos o servicios de calidad. Un estudio de Brymer, Pavesic \& Satisfaction (1990) reveló que, mediante una cultura de servicio de calidad, es posible conservar y atraer a los mejores empleados de la industria. 


\section{LA ESTANDARIZACIÓN Y LA PERSONALIZACIÓN DEL SERVICIO}

La simultaneidad producción-consumo hace posible que, en el área de servicios, las organizaciones puedan ser más flexibles en sus procesos de elaboración que el sector de los productos tangibles. Es así como los servicios pueden ser mejorados sin necesidad de ser rediseñados, modificados infinitamente y personalizados.

Existen grupos de consumidores con sus propias necesidades, deseos y expectativas más o menos similares. De hecho, cada consumidor desea un producto a su medida. Pero esto prácticamente es imposible y demasiado caro. Por esta razón, los consumidores deben hacer concesiones y aceptar la oferta de productos, que de alguna manera, conservan nuestra demanda inicial. Aquí nacen segmentos de consumidores según igualdades y desigualdades que plantean distintos grupos respecto a un mismo producto. Estamos frente a la estandarización de productos.

En el sector servicios, la relación cliente-consumidor es muy estrecha, por tanto, es posible conocer a fondo los requerimientos del cliente, por la prestación de servicios de manera personal. De manera que al ajustar el servicio a los requerimientos específicos del cliente estamos ante una personalización del servicio que brindamos. Por ejemplo, el hecho de llamar por su nombre al huésped de un hotel o al cliente, de un banco, preocuparse de pequeños detalles en las habitaciones del hotel, la venta de una póliza de seguros con prestaciones caracterizadas, preparar algún plato en especial, sorprender al cliente con algún presente por su cumpleaños, etc.

De esta manera es posible afirmar que "en el sector servicio es más fácil elaborar el producto "ideal" deseado por el cliente". Dada la característica del servicio, que implica una vinculación más estrecha entre la empresa y sus clientes, es posible tener conocimiento de sus particulares necesidades y así mejorar constantemente procurando satisfacer las expectativas del cliente. La personalización, finalmente, se traduce en mayores posibilidades de lograr más altos niveles de satisfacción. Entonces, mediante el servicio personalizado es posible lograr, en el largo plazo, una lealtad, y esta, finalmente, se traduce en mayores posibilidades de obtener un rendimiento satisfactorio para la organización. Es en el servicio de hotelería y restaurantes donde mayores son las posibilidades de personalizar el servicio. Por tanto, las probabilidades de obtener más lealtad $\mathrm{y}$ beneficios son inmejorables.

\section{PERSONALIZACIÓN DE SERVICIOS EN LA GESTIÓN HOTELERA}

Muchas empresas trabajan preocupadas por conocer, entender y satisfacer la necesidad de producto o servicio del consumidor: el individuo, el cliente. El objetivo es satisfacer cada necesidad particular a fin de crear una lealtad a largo plazo. En este proceso, la tecnología juega un rol importante para "averiguar quiénes son sus clientes y lo que necesita para asegurar su lealtad" (Berry, Verity, \& otros, s/f).

Según ZOREDA (1996), no hay clientes, sino lo que hay es un cliente, lo que hay es una persona concreta. Afirmación que supone romper la inercia y los moldes del mercado, propiciar un grado cultural distinto sobre la calidad, un grado más allá. Es otra cultura de empresa que busca equilibrar las satisfacciones de los tres grupos de intereses: los accionistas, los clientes y los empleados de una organización.

Para Kotler (2005), la personalización de los servicios es otra ventaja de la mercadotecnia directa. Puede ser expresado de varias maneras: La adaptación de la oferta para que concuerde con las necesidades del mercado meta. Diseño de ofertas exclusivas dirigidas a clientes individuales. Programación de fechas. La personalización de los servicios tiene una ventaja la confidencialidad, debido a que la oferta y la estrategia de la empresa no son visibles para la competencia. La personalización de los servicios permite a las empresas desarrollar una sólida relación con sus clientes, evita que cambien su preferencia por los servicios de los competidores.

Sheraton Club Internacional identifica ocho aspectos importantes que inciden en la venta personal: Identificación y calificación del cliente potencial. Aproximación inicial. Aproximación. Presentación y demostración. Negociación. Superación de las objeciones. Cierre. Seguimiento/ conservación. La creación de cualquier estrategia de servicio implica las siguientes fases: Identificar las necesidades de servicio del cliente y sus expectativas. La creación de grupos de clientes sobre la base de las necesidades y las expectativas. Lo relevante es entender la necesidad de servicio del cliente y luego brindar un servicio, de tal manera 
que haga sentir al cliente que está recibiendo algo que no puede conseguir en otro lugar al mismo precio. Para esto es necesario desarrollar una estrategia de servicio propio.

\section{ELEMENTOS BÁSICOS PARA APLICAR LA PERSONALIZACIÓN DE SERVICIO}

La personalización de servicios exige mucho más que simplemente una buena voluntad. Es un camino sin retorno, involucra a toda la organización y esencialmente exige la creación de atributos que puedan ser percibidos como únicos por el cliente. No obstante, el estilo de gestión participativo y la dirección por objetivos se convierten cada vez más en un elemento decisivo para conseguir la adecuada flexibilidad y capacidad de adaptación a las necesidades personales del cliente, especialmente en el caso de existir varios establecimientos y dispersión geográfica o cualitativa. En la búsqueda de actitud positiva para resolución de problemas, es necesario delegar la capacidad y responsabilidad de la toma de decisiones no solo a nivel de directores de hotel, sino a nivel de jefes de departamentos, e incluso a nivel inferior.

Las empresas hoteleras con varios establecimientos disponen de algún sistema de presupuestos y fijación de objetivos, cuya consecución puede tener, incluso, incidencia a nivel de director o alcanzar a los jefes de departamento. Es frecuente que los responsables dispongan de cierto grado de autonomía en cuanto a inversiones, contratación de personal, incentivos, etc. Sin embargo, en general, estos objetivos se basan en aspectos cuantitativos relacionados con cifras de ventas, rentabilidades, sin tener en cuenta indicadores del grado de satisfacción de los clientes.

Cadenas hoteleras con clara vocación de atención al cliente han comenzado a fijar objetivos de tipo cualitativo, basados en la satisfacción que manifiesta el cliente. Podríamos identificar los requisitos de información solicitados por la dirección de las empresas hoteleras con las distintas fases por las que ha atravesado el turismo en nuestro país. Fuerte crecimiento y expansión de la demanda: información centrada en el índice de ocupación y posiciones de venta. Crisis del sector y recesión de la demanda: preocupación por el control de los costos para asegurar la continuidad o supervivencia. Reactivación del sector: análisis del grado de satisfacción del cliente para asegurar una demanda sostenida con un nivel de rentabilidad adecuado.
Si bien la mayoría de las grandes cadenas hoteleras han asumido perfectamente la necesidad de disponer de información rápida y fiable, tanto en relación con los ingresos como en relación con los costos, e incluso, incorporan sistemas de información fiables para el análisis del grado de satisfacción de los clientes, la gran mayoría de empresas hoteleras pequeñas con hoteles individuales no han llegado más allá de la primera fase. Estas empresas deberían plantearse la necesidad de elaborar un cuadro de mando que incorporase las variables de gestión clave del hotel, además de fijarse solamente en los porcentajes de ocupación.

\section{LAS VENTAJAS COMPETITIVAS EN EL SERVICIO}

El mercado mundial está lleno de casos en que los innovadores, empresas que son primeras en lanzar al mercado un nuevo producto, servicio o proceso, se lamentan del hecho de que imitadores y competidores se benefician más de la innovación que ellas. Esto no deja de ser sorprendente, considerando que las empresas que ingresan al mercado con un nuevo concepto se hacen una ventaja estratégica; sin embargo, es frecuente observar que un rápido seguidor (fast second) y hasta un lento tercero (slow third) pueden superar al innovador haciéndose con los beneficios fabulosos por el desarrollo de nuevos conceptos para satisfacer las necesidades de los consumidores.

\section{LA INNOVACIÓN EN LOS SERVICIOS}

Es una variable estratégica vital en el desarrollo competitivo de las empresas de servicios turísticos que hayan alcanzado el éxito. La innovación dota de fuerza a las organizaciones, permitiendo mejorar su ciclo vital, induce mayor entusiasmo, compromiso e interés dentro de la organización y permite a las empresas de servicios de "ser el primero" captando una posición competitiva duradera. La innovación tecnológica es aprendizaje continuo para mejorar productos, procesos y gestión que incremente productividad y competitividad; es un proceso de aprendizaje de diferentes vías: imitando, haciendo, interactuando con otros agentes, así como investigando.

Según Hamel (1998), en un mundo discontinuo la innovación estratégica es la clave de la creación de la riqueza. La innovación estratégica es la capacidad de concebir el modelo actual de negociar, de forma que cree un nuevo valor para los clientes, deje fuera a competidores y produzca nueva ri- 
queza para todos los participantes en la empresa. El impacto de la ciencia en la industria moderna es grandioso; en la actualidad en los países desarrollados, la investigación ha ocasionado cambios rápidos, debido a los recursos utilizados en estas actividades, y que depende de factores como la competencia, la necesidad de sustitutos, etc. Sin embargo, esta gestión es más importante para la supervivencia y el crecimiento de más empresas.

La creación -invención- se centra en la idea, abarca todo el proceso por el cual la idea nueva se lleva a un uso productivo. La innovación puede definirse: Entrada inicial al mercado de un producto, proceso o servicio, de un nuevo invento. Cuando una empresa produce, usa métodos o insumos nuevos para ella, realizando cambios.

\section{MÉTODOS}

Se realizó una investigación exploratoria porque no existen estudios previos sobre el impacto de la personalización de los servicios en la empresa hotelera. Con elementos descriptivos, diseño no experimental, se pretende observar el fenómeno, la realidad, sin manipular las variables transeccional descriptivas con el propósito de describir variables y analizar su incidencia en un determinado tiempo. El universo empresas hoteleras, población de 50 por método de muestreo aleatorio simple, que dio 24 hoteles. Los datos se recogieron entrevistando a 384 clientes en hoteles, en el momento de cancelar. Como técnica se usó el cuestionario diseñado con 21 interrogantes: 15 preguntas cerradas de respuesta múltiple, dos preguntas semicerradas con libertad al entrevistado de incluir alguna opción y cuatro preguntas abiertas, ya que las posibles respuestas podían ampliarse. Siendo la pregunta $\mathrm{N}^{\circ} 7$ relevante porque dará información necesaria para apoyar o negar la hipótesis. Se hizo el análisis con estadística descriptiva y la presentación se realizó con gráficos circulares.

\section{RESULTADOS}

De acuerdo a la pregunta sobre conocimiento cabal del significado y práctica de personalización de servicios para cada empresa encuestada, se expresa en gráfico la percepción que tienen las empresas hoteleras respecto a esta estrategia en estudio.

\section{¿En qué consiste el servicio personalizado?}

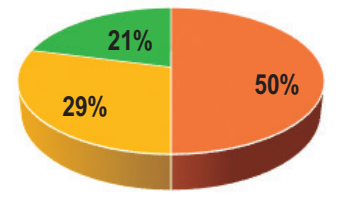

\footnotetext{
- Atención personal " Anticipa a pedidos

- Detalles personales
}

Se deduce que 12 empresas hoteleras encuestadas (50\%) manifiestan que la personalización de servicios está en la atención personal al cliente del hotel. La anticipación a los pedidos del cliente, mediante una base de datos, es lo más relevante dentro de su estrategia de personalización de servicios para siete empresas (29\%), y cinco empresas $(21 \%)$ consideran la inclusión de detalles personales en las habitaciones.

La pregunta sobre cuáles fueron los beneficios logrados por las empresas encuestadas, en el gráfico se observa que para 12 empresas la aplicación del servicio personalizado se traduce en un aumento sustancial de sus ventas. Sorprende observar que para seis empresas (25\%) el uso de esta herramienta implicó un aumento de imagen de la empresa hotelera; asimismo, cinco empresas encuestadas aseguraron haber logrado un mejor servicio al cliente, y, apenas un $4 \%$ de la muestra habrían logrado fidelizar al cliente mediante la aplicación del servicio personalizado.

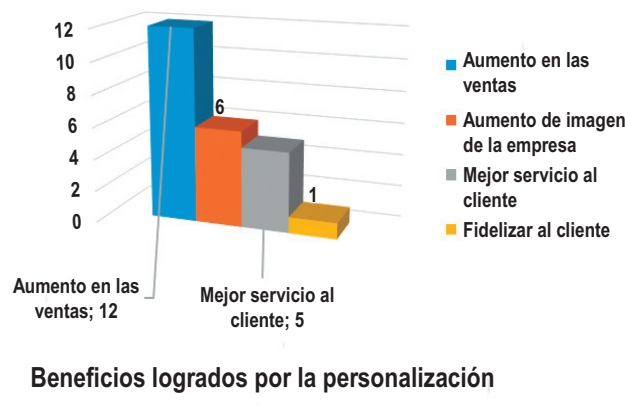

Para 13 empresas, 54\% de la muestra, la fidelidad del cliente es el mejor indicador de satisfacción alcanzada por sus clientes, respecto a los servicios esperados y obtenidos. Para nueve empresas, $39 \%$ de la muestra, el incremento de los retornos es el indicador que mejor expresa la satisfacción alcanzada por sus clientes. Dos de las empresas encuestadas afirman que la ocupa- 
bilidad alcanzada es el reflejo de la satisfacción de los clientes respecto a los servicios ofertados por la empresa.

\section{DISCUSIÓN}

Con los datos obtenidos del cuestionario aplicado a clientes y de las entrevistas con los gerentes generales, se probó la hipótesis planteada: con el coeficiente de correlación de 0.96 , dio un 0.93 , que se interpretó como la variación de la Rentabilidad se explica en un $93 \%$ por la variación del servicio personalizado, medido a través del nivel de ventas. Existe alta relación entre estas variables. Significa que en la medida que mejore el servicio de manera personalizada, se espera un incremento en las ventas que, a su vez, influya en el incremento de la rentabilidad. Para la prueba de hipótesis se aplicó la prueba estadística chi cuadrado, la hipótesis nula H0: Rentabilidad y servicio personalizado son independientes y la hipótesis alterna $\mathrm{H} 1$ : Rentabilidad y servicio personalizado son variables dependientes. Al combinar las respuestas relacionadas con el grado de satisfacción de los clientes y el nivel de rentabilidad alcanzado por el negocio, se obtuvo el siguiente cuadro: Satisfacción en el servicio y su efecto en la rentabilidad

\begin{tabular}{cccc} 
Satisfacción & Mejoró rentabilidad & $\begin{array}{c}\text { No mejoró } \\
\text { rentabilidad }\end{array}$ & Total \\
\hline Bastante & 265 & 10 & 275 \\
\hline Regular & 45 & 15 & 60 \\
\hline Poco & 13 & 25 & 38 \\
\hline Ninguno & 6 & 5 & 11 \\
\hline Total & 329 & 55 & 384
\end{tabular}

La prueba chi cuadrado dio $t=7.81$; Con $\alpha$ $=0.05$, y aplicando la fórmula a los datos, se obtiene X2 c $=121.9$. Por lo que se rechazó la hipótesis nula y se aceptó la hipótesis alternativa, que concuerda con el análisis de correlación. En consecuencia, de acuerdo a los datos obtenidos en la presente investigación podemos afirmar que: El servicio personalizado permite alcanzar una mayor rentabilidad.

Para la hipótesis específica 1 , se midió la relación existente entre los incrementos de inversión en términos porcentuales y los niveles de disminución de los costos. En la medida que el personal tiene mayor capacitación, se espera una mejora en la eficiencia; por tanto, disminución de costos. Estos resultados se observan en el cuadro de la relación entre capacitación y disminución de costos.

\begin{tabular}{cc}
$\%$ Inversión & Disminución \\
\hline Capacitación & Costos $(\%)$ \\
\hline 10 & -4 \\
\hline 20 & -8 \\
\hline 30 & -12 \\
\hline 40 & -15 \\
\hline 50 & -18
\end{tabular}

Realizando el análisis de correlación, se obtiene un coeficiente de determinación con un 0.99; como se podrá apreciar, es una relación casi perfecta. La tabla que muestra los datos para probar la hipótesis corresponde a la relación entre inversión y disminución de costos.

\begin{tabular}{cccc}
\multicolumn{4}{c}{ Disminución Costos } \\
\hline Inv. Capacitación & Alto & Bajo & Total \\
\hline Baja $(-10 \%)$ & 27 & 56 & 83 \\
\hline Media $(11-29 \%)$ & 87 & 49 & 136 \\
\hline Alta $(+30 \%)$ & 153 & 12 & 165 \\
\hline Total & 267 & 117 & 384
\end{tabular}

La prueba chi cuadrado dio $\mathrm{t} X 2 \mathrm{t}=5.99$ con $\alpha=0.05$, y aplicando la fórmula a los datos, se obtiene X2 c $=97.52$. Por lo que se rechazó la hipótesis nula aceptando la hipótesis alternativa, que concuerda con al análisis de correlación. Por tanto, de acuerdo a los datos obtenidos en la presente investigación podemos afirmar que: El desarrollo de una cultura de servicio personalizado al cliente eleva la competitividad.

\section{CONCLUSIONES}

1. La personalización de los servicios asegura mejor satisfacción del cliente y alta rentabilidad a la empresa hotelera, y el nivel de conocimiento que tienen las empresas hoteleras de Lima sobre la estrategia de personalización de los servicios es alta. Por tanto, un alto porcentaje de clientes recomendaría el servicio.

2. El grado de satisfacción alcanzado por las empresas en el uso de la estrategia de personalización de los servicios es importante, por lo que declararon estar muy satisfechas. Los requisitos básicos para desarrollar 
estrategias de personalizar los servicios en las empresas hoteleras son: tecnología adecuada, capacidad económica, personal altamente calificado y una política de servicios orientada al cliente, entre otros.

3. Entre las variables que contribuyen al éxito de las empresas hoteleras resalta la calidad del personal. El factor humano es determinante, sobre todo, a la hora de aplicar un servicio personalizado. Entre los mecanismos que contribuyen al desarrollo de políticas dirigidas a personalizar los servicios hoteleros están básicamente la búsqueda de aumento de ventas.Indicaron que el pedido de los clientes es la razón fundamental de la personalización de los servicios.

\section{REFERENCIAS BIBLIOGRÁFICAS}

Agut Nieto, S., Grandio Botella, A., \& Peris Pichastor , R. (s/f). Los recursos humanos en el Ámbito turístico. Madrid Síntesis S.A.

Andersen, A. (1995). La calidad en España: La calidad en el Ámbito del Bien de Consumo y los Servicios. Madrid: Argentaria.

Berry, J., Verity, j., \& otros. (s/f). Mercadotecnia de Base de Datos: Un Nuevo Instrumento Poderoso para las Ventas. En Mercadotecnia de Servicios de Lovelock c.

Carrasco Fernandez , S. (2013). Comunicación y atención al cliente en hostelería y turismo. España: Paraninfo.

Czinkota, M. R., \& Ronkainen, I. A. (2007). Marketing internacional. México: Thomson/ South-Western.

Daza Higuera, J. M. (2013). Análisis de la medición de calidad en los servicios hoteleros. Bogotá -Colombia: Criterio Libre.

Gallego, J. F. (2006). Gestión de Alimentos y Bebidas Para Hoteles, Bares y Restaurantes. Madrid: Paraninfo.

Gallego, J. F. (2013). Marketing para Hoteles y Restaurantes en los nuevos escenarios. Madrid: Paraninfo.

Hamel, G. (1998). Revista Harvard. Deusto.

Kotler, P. (2005). Las Preguntas más frecuentes sobre Marketing. Bogotá. Colombia: Norma $1^{\circ}$ Edición.

Porter, M. E. (2010). Ventaja Competitiva: Creación $y$ sostenimiento de un desarrollo superior. España: Pirámide.

Schnarch, A. (1996). Innovación Tecnológica y Éxito Empresarial. Barcelona: Ariel. 